\title{
On $k$-Fibonacci hybrid numbers and their matrix representations
}

\author{
Fügen Torunbalcı Aydın \\ Yildiz Technical University, Faculty of Chemical and Metallurgical Engineering \\ Department of Mathematical Engineering \\ Davutpasa Campus, 34220, Esenler, Istanbul, Turkey \\ e-mails: faydin@yildiz.edu.tr, ftorunay@gmail.com
}

Received: 7 December 2020 Revised:. 17 September 2021 Accepted: 21 September 2021

\begin{abstract}
In this paper, $k$-Fibonacci hybrid numbers are defined. Also, some algebraic properties of $k$-Fibonacci hybrid numbers such as Honsberger identity, Binet Formula, generating functions, d'Ocagne identity, Cassini and Catalan identities are investigated. In addition, we also give $2 \times 2$ and $4 \times 4$ representations of the $k$-Fibonacci hybrid numbers $H F_{k, n}$.
\end{abstract}

Keywords: Hybrid number, $k$-Fibonacci number, $k$-Fibonacci hybrid number, $k$-Fibonacci number hybrid matrix.

2020 Mathematics Subject Classification: 11B37, 11B39, 11B50, 11C20, 15A33.

\section{Introduction}

The hybrid number system can be accepted as a generalization of the complex, dual and hyperbolic number systems. In 2018, firstly, set of hybrid numbers was introduced by Özdemir [9] as follows:

$$
\mathbb{K}=\left\{a+b i+c \varepsilon+d h \mid a, b, c, d \in \mathbb{R}, i^{2}=-1, \varepsilon^{2}=0, h^{2}=1\right\},
$$

where units satisfy the rules

$$
i h=-h i=\varepsilon+i
$$

The set $\mathbb{K}$ of hybrid numbers forms non-commutative ring with respect to the addition and multiplication operations.

Taking two hybrid numbers $z_{1}=a_{1}+b_{1} i+c_{1} \varepsilon+d_{1} h, z_{2}=a_{2}+b_{2} i+c_{2} \varepsilon+d_{2} h$ and $s \in \mathbb{R}$ get: 
- Equality $z_{1}=z_{2}$, if and only if, $a_{1}=a_{2}, b_{1}=b_{2}, c_{1}=c_{2}$, and $d_{1}=d_{2}$;

- $\operatorname{Sum} z_{1}+z_{2}=\left(a_{1}+a_{2}\right)+\left(b_{1}+b_{2}\right) i+\left(c_{1}+c_{2}\right) \varepsilon+\left(d_{1}+d_{2}\right) h$;

- Subtraction $z_{1}-z_{2}=\left(a_{1}-a_{2}\right)+\left(b_{1}-b_{2}\right) i+\left(c_{1}-c_{2}\right) \varepsilon+\left(d_{1}-d_{2}\right) h$;

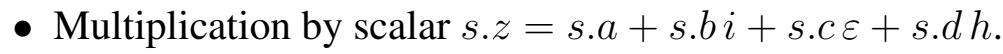

The real number $C(z)=z \cdot \bar{z}=\bar{z} \cdot z=a^{2}+(b-c)^{2}-c^{2}-d^{2}$ is called the character of the hybrid number $z$. A new expression for the character of a hybrid number $z$ is given by

$$
C(z)=(a-b)^{2}-2 b(c-a)-d^{2}
$$

The hybrid matrix corresponding to the hybrid number $z$ is given by following $2 \times 2$ matrix

$$
A=\left(\begin{array}{cc}
a+c & b-c+d \\
c-b+d & a-c
\end{array}\right) .
$$

For more details on hybrid numbers, see [2,9].

In 2007, the $k$-Fibonacci sequence $\left\{F_{k, n}\right\}_{n \in \mathbb{N}}$ is defined by Falcon and Plaza [4-8] as follows

$$
\left\{\begin{aligned}
F_{k, 0}= & 0, F_{k, 1}=1 \\
F_{k, n+1}= & k F_{k, n}+F_{k, n-1}, n \geq 1 \\
o r & \\
\left\{F_{k, n}\right\}_{n \in \mathbb{N}}= & \left\{0,1, k, k^{2}+1, k^{3}+2 k, k^{4}+3 k^{2}+1, \ldots\right\}
\end{aligned}\right.
$$

Here, $k$ is a positive real number.

In 2015, Ramirez [10] defined the the $k$-Fibonacci and the $k$-Lucas quaternions. For more details about these quaternions see, for example, [1,10,11].

In 2018, Cerda-Morales [3] defined the generalized hybrid Fibonacci numbers.

The aim of this study is to define $k$-Fibonacci hybrid numbers and give their algebraic properties by using hybrid numbers based on Özdemir's study in [9].

\section{The $k$-Fibonacci hybrid numbers}

In this section, firstly the $k$-Fibonacci hybrid numbers will be defined as follows:

$$
H F_{k, n}=F_{k, n}+i F_{k, n+1}+\varepsilon F_{k, n+2}+h F_{k, n+3}
$$

where units satisfy the rules as follows

$$
i^{2}=-1, \varepsilon^{2}=0, h^{2}=1, i h=-h i=i+\varepsilon, \quad \varepsilon i=1+h, i \varepsilon=1-h, \varepsilon h=-h \varepsilon=\varepsilon .
$$

where $h i+\varepsilon+i=0$.

Similarly, the $k$-Lucas hybrid numbers will be defined as follows:

$$
H L_{k, n}=L_{k, n}+i L_{k, n+1}+\varepsilon L_{k, n+2}+h L_{k, n+3}
$$

where (5) conditions are satisfied. 
The $k$-Fibonacci hybrid sequence $H F_{k, n}$ satisfies the recurrence relationship

$$
H F_{k, n}=k H F_{k, n-1}+H F_{k, n-2} \text {. }
$$

with initial conditions

$$
\begin{aligned}
& H F_{k, 0}=i+\varepsilon k+h\left(k^{2}+1\right), \text { and } \\
& H F_{k, 1}=1+i k+\varepsilon\left(k^{2}+1\right)+h\left(k^{3}+2 k\right) .
\end{aligned}
$$

Similarly, The $k$-Lucas hybrid sequence $H L_{k, n}$ satisfies the recurrence relationship

$$
H L_{k, n}=k H L_{k, n-1}+H L_{k, n-2} \text {. }
$$

with initial conditions

$$
\begin{array}{r}
H L_{k, 0}=2+k i+\varepsilon\left(k^{2}+2\right)+h\left(k^{3}+3 k\right), \text { and } \\
H L_{k, 1}=k+i\left(k^{2}+2\right)+\varepsilon\left(k^{3}+3 k\right)+h\left(k^{4}+4 k^{2}+2\right) .
\end{array}
$$

Taking into account (1), the norm and character of a $k$-Fibonacci hybrid number is given as

$$
\left\|H F_{k, n}\right\|^{2}=C\left(H F_{k, n}\right)
$$

where $C\left(H F_{k, n}\right)=\left(F_{k, n}-F_{k, n+1}\right)^{2}-2 F_{k, n+1}\left(F_{k, n+2}-F_{k, n}\right)-F_{k, n+3}^{2}$.

Let $H F_{k, n}$ and $H F_{k, m}$ be two $k$-Fibonacci hybrid numbers. The addition and subtraction of two $k$-Fibonacci hybrid numbers are defined in the obvious way,

$$
\begin{aligned}
H F_{k, n} \pm H F_{k, m}= & \left(F_{k, n}+i F_{k, n+1}+\varepsilon F_{k, n+2}+h F_{k, n+3}\right) \\
& \pm\left(F_{k, m}+i F_{k, m+1}+\varepsilon F_{k, m+2}+h F_{k, m+3}\right) \\
= & \left(F_{k, n} \pm F_{k, m}\right)+i\left(F_{k, n+1} \pm F_{k, m+1}\right) \\
& +\varepsilon\left(F_{k, n+2} \pm F_{k, m+2}\right)+h\left(F_{k, n+3} \pm F_{k, m+3}\right) .
\end{aligned}
$$

Accordingly, we will use table below (Table 1) for the multiplication of any two hybrid numbers. This table shows us that the multiplication operation in the hybrid numbers is not commutative. But it has the property of associativity.

\begin{tabular}{|r||r|r|r|r|}
\hline$x$ & 1 & $i$ & $\varepsilon$ & $h$ \\
\hline \hline 1 & 1 & $i$ & $\varepsilon$ & $h$ \\
$i$ & $i$ & -1 & $1-h$ & $\varepsilon+i$ \\
$\varepsilon$ & $\varepsilon$ & $1+h$ & 0 & $-\varepsilon$ \\
$h$ & $h$ & $-\varepsilon-i$ & $\varepsilon$ & 1 \\
\hline
\end{tabular}

Table 1: Multiplication scheme of hybrid numbers [1]

Multiplication of two $k$-Fibonacci hybrid numbers, which are non-commutative is defined by

$$
\begin{aligned}
H F_{k, n} \times H F_{k, m}= & {\left[F_{k, n} F_{k, m}-F_{k, n+1} F_{k, m+1}-F_{k, n+2} F_{k, m+2}+F_{k, n+3} F_{k, m+3}\right] } \\
& +i\left[F_{k, n} F_{k, m+1}+F_{k, n+1} F_{k, m}-F_{k, n+2} F_{k, m+3}-F_{k, n+3} F_{k, m+2}\right] \\
& +\varepsilon\left[F_{k, n} F_{k, m+2}-F_{k, n+1} F_{k, m+3}+F_{k, n+2} F_{k, m}-F_{k, n+3} F_{k, m+1}\right] \\
& +h\left[F_{k, n} F_{k, m+3}+F_{k, n+1} F_{k, m+2}+F_{k, n+2} F_{k, m+1}+F_{k, n+3} F_{k, m}\right] \\
\neq & H F_{k, m} \times H F_{k, n} .
\end{aligned}
$$


The conjugation of the $k$-Fibonacci hybrid number is defined as follows

$$
\overline{H F}_{k, n}=F_{k, n}-i F_{k, n+1}-\varepsilon F_{k, n+2}-h F_{k, n+3},
$$

Therefore, the norm of the $k$-Fibonacci hybrid number $H F_{k, n}$ is defined as follows

$$
\begin{aligned}
\left\|H F_{k, n}\right\|^{2} & =H F_{k, n} \cdot \overline{H F}_{k, n} \\
& =F_{k, n}^{2}+F_{k, n+1}^{2}-F_{k, n+3}^{2}-2 F_{k, n+1} F_{k, n+2} .
\end{aligned}
$$

In the following theorem, some properties related to the $k$-Fibonacci hybrid numbers are given.

Theorem 1. Let $H F_{k, n}$ be the $k$-Fibonacci hybrid number. In this case, we can give the following relations:

$$
\begin{gathered}
H F_{k, n+2}=k H F_{k, n+1}+H F_{k, n}, \\
H L_{k, n}=H F_{k, n+1}+H F_{k, n-1}, \\
k H L_{k, n}=H F_{k, n+2}-H F_{k, n-2} .
\end{gathered}
$$

Proof: (14): By using (4) we get,

$$
\begin{aligned}
H F_{k, n}+k H F_{k, n+1}= & \left(F_{k, n}+k F_{k, n+1}\right)+i\left(F_{k, n+1}+k F_{k, n+2}\right) \\
& +\varepsilon\left(F_{k, n+2}+k F_{k, n+3}\right)+h\left(F_{k, n+3}+k F_{k, n+4}\right) \\
= & F_{k, n+2}+i F_{k, n+3}+\varepsilon F_{k, n+4}+h F_{k, n+5} \\
= & H F_{k, n+2}
\end{aligned}
$$

(15): By using (4) we get,

$$
\begin{aligned}
H F_{k, n+1}+H F_{k, n-1}= & \left(F_{k, n+1}+F_{k, n-1}\right)+i\left(F_{k, n+2}+F_{k, n}\right) \\
& +\varepsilon\left(F_{k, n+3}+F_{k, n+1}\right)+h\left(F_{k, n+4}+F_{k, n+2}\right) \\
= & L_{k, n}+i L_{k, n+1}+\varepsilon L_{k, n+2}+h L_{k, n+3} \\
= & H L_{k, n}
\end{aligned}
$$

(16): By using (4) we get,

$$
\begin{aligned}
H F_{k, n+2}-H F_{k, n-2}= & \left(F_{k, n+2}-F_{k, n-2}\right)+i\left(F_{k, n+3}-F_{k, n-1}\right) \\
& +\varepsilon\left(F_{k, n+4}-F_{k, n}\right)+h\left(F_{k, n+5}+F_{k, n+1}\right) \\
= & k\left[L_{k, n}+i L_{k, n+1}+\varepsilon L_{k, n+2}+h L_{k, n+3}\right] \\
= & k H L_{k, n}
\end{aligned}
$$

where $F_{k, n+1}+F_{k, n-1}=L_{k, n}$ and $F_{k, n+2}-F_{k, n-2}=k L_{k, n}$ are used.

Theorem 2 (Honsberger identity). For $n, m \geq 0$ the Honsberger identity for the $k$-Fibonacci hybrid numbers $H F_{k, n}$ and $H F_{k, m}$ is given by

$$
\begin{aligned}
H F_{k, n} H F_{k, m}+H F_{k, n+1} H F_{k, m+1}= & H F_{k, n+m+1}+\left(-F_{k, n+m+3}+2 F_{k, n+m+4}\right. \\
& \left.+F_{k, n+m+7}\right)+F_{k, n+m+2} i+F_{k, n+m+3} \varepsilon \\
& +F_{k, n+m+4} h .
\end{aligned}
$$


Proof: (17): By using (4) we get,

$$
\begin{array}{rl}
H F_{k, n} & H F_{k, m}+H F_{k, n+1} H F_{k, m+1} \\
= & \left(F_{k, n} F_{k, m}+F_{k, n+1} F_{k, m+1}\right)-\left(F_{k, n+1} F_{k, m+1}+F_{k, n+2} F_{k, m+2}\right) \\
& +\left(F_{k, n+3} F_{k, m+3}+F_{k, n+4} F_{k, m+4}\right)+\left(F_{k, n+1} F_{k, m+2}+F_{k, n+2} F_{k, m+2}\right) \\
& \left.+\left(F_{k, n+2} F_{k, m+1}+F_{k, n+3} F_{k, m+1}\right)\right] \\
+ & i\left[\left(F_{k, n} F_{k, m+1}+F_{k, n+1} F_{k, m+2}\right)+\left(F_{k, n+1} F_{k, m}-F_{k, n+2} F_{k, m+1}\right)\right. \\
& \left.+\left(F_{k, n+1} F_{k, m+3}+F_{k, n+2} F_{k, m+4}\right)-\left(F_{k, n+3} F_{k, m+1}+F_{k, n+4} F_{k, m+2}\right)\right] \\
+ & \varepsilon\left[\left(F_{k, n} F_{k, m+2}+F_{k, n+1} F_{k, m+3}\right)+\left(F_{k, n+2} F_{k, m}+F_{k, n+3} F_{k, m+1}\right)\right. \\
& \left.+\left(F_{k, n+1} F_{k, m+3}+F_{k, n+2} F_{k, m+4}\right)-\left(F_{k, n+3} F_{k, m+1}+F_{k, n+4} F_{k, m+2}\right)\right] \\
& \left.\left.-\left(F_{k, n+2} F_{k, m+3}+F_{k, n+3} F_{k, m+4}\right)\right]+\left(F_{k, n+3} F_{k, m+2}+F_{k, n+4} F_{k, m+3}\right)\right] \\
+ & h\left[\left(F_{k, n} F_{k, m+3}+F_{k, n+1} F_{k, m+4}\right)+\left(F_{k, n+3} F_{k, m}+F_{k, n+4} F_{k, m+1}\right)\right. \\
- & \left.\left(F_{k, n+1} F_{k, m+2}+F_{k, n+2} F_{k, m+3}\right)+\left(F_{k, n+2} F_{k, m+1}+F_{k, n+3} F_{k, m+2}\right)\right] \\
= & \left(F_{k, n+m+1}-F_{k, n+m+3}+2 F_{k, n+m+4}+F_{k, n+m+7}\right) \\
+ & 2\left[F_{k, n+m+2} i+F_{k, n+m+3} \varepsilon+F_{k, n+m+4} h\right] \\
= & H F_{k, n+m+1}+\left(-F_{k, n+m+3}+2 F_{k, n+m+4}+F_{k, n+m+7}\right) \\
+ & F_{k, n+m+2} i+F_{k, n+m+3} \varepsilon+F_{k, n+m+4} h . \\
&
\end{array}
$$

where the identity $F_{k, n} F_{k, m}+F_{k, n+1} F_{k, m+1}=F_{k, n+m+1}$ was used [5].

Theorem 3 (Generating function). Let $H F_{k, n}$ be the $k$-Fibonacci hybrid number. For the generating function for these numbers is as follows:

$$
g_{H F_{k, n}}(t)=\sum_{s=1}^{n} H F_{k, n} t^{n}=\frac{H F_{k, 0}+\left(H F_{k, 1}-k H F_{k, 0}\right) t}{1-k t-t^{2}}
$$

Proof: Using the definition of generating function, we obtain

$$
g_{H F_{k, n}}(t)=H F_{k, 0}+H F_{k, 1} t+\cdots+H F_{k, n} t^{n}+\cdots .
$$

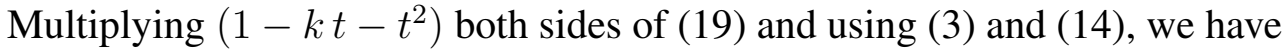

$$
\begin{aligned}
\left(1-k t-t^{2}\right) g_{H F_{k, n}}(t)= & H F_{k, 0}+t\left(H F_{k, 1}-k H F_{k, 0}\right) \\
& +t^{2}\left(H F_{k, 2}-k H F_{k, 1}-H F_{k, 0}\right)+\cdots \\
& +t^{n+1}\left(H F_{k, n+1}-k H F_{k, n}-H F_{k, n-1}\right)+\cdots \\
= & H F_{k, 0}+t\left(H F_{k, 1}-k H F_{k, 0}\right)+0+0+\cdots
\end{aligned}
$$

where

$$
\begin{aligned}
-k t g_{H F_{k, n}}(t) & =-k t H F_{k, 0}-k t^{2} H F_{k, 1}-\ldots-k t^{n+1} H F_{k, n}-\cdots, \\
-t^{2} g_{H F_{k, n}}(t) & =-t^{2} H F_{k, 0}-t^{3} H F_{k, 1}-\cdots-t^{n+1} H F_{k, n-1}-\cdots
\end{aligned}
$$

and

$$
H F_{k, n+1}-k H F_{k, n}-H F_{k, n-1}=0
$$

are used. Thus, the proof is completed. 
Theorem 4 (Binet's Formula). Let $H F_{k, n}$ be the $k$-Fibonacci hybrid number. For $n \geq 1$, Binet's formula for these numbers is as follows:

$$
H F_{k, n}=\frac{1}{\alpha-\beta}\left(\hat{\alpha} \alpha^{n}-\hat{\beta} \beta^{n}\right)
$$

where

$$
\begin{gathered}
\hat{\alpha}=1+(k-\beta) i+\left(k^{2}-k \beta+1\right) \varepsilon+\left(k^{3}-\left(k^{2}+1\right) \beta+2 k\right) h \\
=1+i \alpha+\varepsilon \alpha^{2}+h \alpha^{3}, \\
-\hat{\beta}=1+(k-\alpha) i+\left(k^{2}-\alpha k+1\right) \varepsilon+\left(k^{3}-\left(k^{2}+1\right) \alpha+2 k\right) h \\
=1+i \beta+\varepsilon \beta^{2}+h \beta^{3}, \\
\alpha=\frac{k+\sqrt{k^{2}+4}}{2}, \beta=\frac{k-\sqrt{k^{2}+4}}{2}, \alpha+\beta=k, \quad \alpha-\beta=\sqrt{k^{2}+4}, \quad \alpha \beta=-1 .
\end{gathered}
$$

Proof: Using the Binet formula for $k$-Fibonacci number [5], we obtain

$$
\begin{aligned}
H F_{k, n} & =F_{k, n}+i F_{k, n+1}+\varepsilon F_{k, n+2}+h F_{k, n+3} \\
& =\frac{\alpha^{n}-\beta^{n}}{\sqrt{k^{2}+4}}+i\left(\frac{\alpha^{n+1}-\beta^{n+1}}{\sqrt{k^{2}+4}}\right)+\varepsilon\left(\frac{\alpha^{n+2}-\beta^{n+2}}{\sqrt{k^{2}+4}}\right)+h\left(\frac{\alpha^{n+3}-\beta^{n+3}}{\sqrt{k^{2}+4}}\right) \\
& =\frac{\alpha^{n}\left(1+i \alpha+\varepsilon \alpha^{2}+h \alpha^{3}\right)-\beta^{n}\left(1+i \beta+\varepsilon \beta^{2}+h \beta^{3}\right)}{\sqrt{k^{2}+4}} \\
& =\frac{1}{\sqrt{k^{2}+4}}\left(\hat{\alpha} \alpha^{n}-\hat{\beta} \beta^{n}\right) .
\end{aligned}
$$

where $\hat{\alpha}=1+i \alpha+\varepsilon \alpha^{2}+h \alpha^{3}, \hat{\beta}=1+i \beta+\varepsilon \beta^{2}+h \beta^{3}$.

Theorem 5 (d'Ocagne's identity). For $n, m \geq 0$ the D'Ocagne's identity for the $k$-Fibonacci hybrid numbers $H F_{k, n}$ and $H F_{k, m}$ is given by

$$
\begin{aligned}
H F_{k, n} H F_{k, m+1}-H F_{k, n+1} H F_{k, m}= & (-1)^{m}\left[(1-k) F_{k, n-m}\right. \\
& +k\left(F_{k, n-m}+L_{k, n-m}\right) i+\left(L_{k, n-m}\right) \varepsilon \\
& \left.+\left(3 k F_{k, n-m}+\left(k^{2}-1\right) L_{k, n-m}\right) h\right] \\
\text { or } \quad & \\
= & \frac{(-1)^{m} \hat{\alpha} \hat{\beta}\left(\alpha^{n-m}-\beta^{n-m}\right)}{\alpha-\beta} .
\end{aligned}
$$

Proof: (21) By using (4) we get,

$$
\begin{aligned}
H & F_{k, n} H F_{k, m+1}-H F_{k, n+1} H F_{k, m} \\
= & \left(F_{k, n} F_{k, m+1}-F_{k, n+1} F_{k, m}\right)-\left(F_{k, n+1} F_{k, m+2}-F_{k, n+2} F_{k, m+1}\right) \\
& +\left(F_{k, n+3} F_{k, m+4}-F_{k, n+4} F_{k, m+3}\right)+\left(F_{k, n+1} F_{k, m+3}-F_{k, n+2} F_{k, m+2}\right) \\
& \left.+\left(F_{k, n+2} F_{k, m+2}-F_{k, n+3} F_{k, m+1}\right)\right] \\
+ & i\left[\left(F_{k, n} F_{k, m+2}-F_{k, n+1} F_{k, m+1}\right)+\left(F_{k, n+1} F_{k, m+1}-F_{k, n+2} F_{k, m}\right)\right. \\
& \left.-\left(F_{k, n+3} F_{k, m+2}-F_{k, n+4} F_{k, m+1}\right)+\left(F_{k, n+1} F_{k, m+4}-F_{k, n+2} F_{k, m+3}\right)\right] \\
+ & \varepsilon\left[\left(F_{k, n} F_{k, m+3}-F_{k, n+1} F_{k, m+2}\right)+\left(F_{k, n+2} F_{k, m+1}-F_{k, n+3} F_{k, m}\right)\right. \\
& +\left(F_{k, n+1} F_{k, m+4}-F_{k, n+2} F_{k, m+3}\right)-\left(F_{k, n+3} F_{k, m+2}-F_{k, n+4} F_{k, m+1}\right) \\
& \left.-\left(F_{k, n+2} F_{k, m+4}-F_{k, n+3} F_{k, m+3}\right)+\left(F_{k, n+3} F_{k, m+3}-F_{k, n+4} F_{k, m+2}\right)\right] \\
+ & h\left[\left(F_{k, n} F_{k, m+4}-F_{k, n+1} F_{k, m+3}\right)+\left(F_{k, n+3} F_{k, m+1}-F_{k, n+4} F_{k, m}\right)\right. \\
& \left.+\left(F_{k, n+2} F_{k, m+2}-F_{k, n+3} F_{k, m+1}\right)-\left(F_{k, n+1} F_{k, m+3}-F_{k, n+2} F_{k, m+2}\right)\right] \\
(- & 1)^{m}\left[(1-k) F_{k, n-m}+k\left(F_{k, n-m}+L_{k, n-m}\right) i+\left(L_{k, n-m}\right) \varepsilon\right. \\
+ & \left.\left(3 k F_{k, n-m}+\left(k^{2}-1\right) L_{k, n-m}\right) h\right],
\end{aligned}
$$

where the identity $F_{k, n} F_{k, m+1}-F_{k, n+1} F_{k, m}=(-1)^{m} F_{k, n-m}$ is used [5]. 
We calculate (21) again with a second method. By using (20) we get

$$
\begin{aligned}
H F_{k, n} H F_{k, m+1}-H F_{k, n+1} H F_{k, m}= & \begin{array}{c}
\left(\frac{\hat{\alpha} \alpha^{n}-\hat{\beta} \beta^{n}}{\alpha-\beta}\right)\left(\frac{\hat{\alpha} \alpha^{m+1}-\hat{\beta} \beta^{m+1}}{\alpha-\beta}\right) \\
-\left(\frac{\hat{\alpha} \alpha^{n+1}-\hat{\beta} \beta^{n+1}}{\alpha-\beta}\right)\left(\frac{\hat{\alpha} \alpha^{m}-\hat{\beta} \beta^{m}}{\alpha-\beta}\right)
\end{array} \\
= & \frac{-\hat{\alpha} \hat{\beta}(\alpha \beta)^{-m} \alpha^{n} \beta^{m+1}-\hat{\beta} \hat{\alpha}(\alpha \beta)^{-m} \beta^{n} \alpha^{m+1}}{(\alpha-\beta)^{2}(\alpha \beta)^{-m}} \\
& +\frac{\hat{\alpha} \hat{\beta}(\alpha \beta)^{-m} \alpha^{n+1} \beta^{m}+\hat{\beta} \hat{\alpha}(\alpha \beta)^{-m} \beta^{n+1} \alpha^{m}}{(\alpha-\beta)^{2}(\alpha \beta)^{-m}} \\
= & \frac{-\hat{\alpha} \hat{\beta} \alpha^{n-m}(\alpha-\beta)-\hat{\beta} \hat{\alpha} \beta^{n-m}(\alpha-\beta)}{(\alpha-\beta)^{2}(\alpha \beta)^{-m}} \\
= & \frac{(-1)^{m} \hat{\alpha} \hat{\beta}\left(\alpha^{n-m}-\beta^{n-m}\right)}{\alpha-\beta}
\end{aligned}
$$

where $\hat{\alpha}=1+i \alpha+\varepsilon \alpha^{2}+h \alpha^{3}$ and $-\hat{\beta}=1+i \beta+\varepsilon \beta^{2}+h \beta^{3}$.

Theorem 6 (Cassini's Identity). Let $H F_{F_{k, n}}$ be the $k$-Fibonacci hybrid number. For $n \geq 1$, Cassini's identity for $H F_{k, n}$ is as follows:

$$
\begin{aligned}
H F_{k, n-1} H F_{k, n+1}-\left(H F_{k, n}\right)^{2} & =(-1)^{n}\left[(1-k)+k(1-k) i+(2-k) \varepsilon+\left(k^{3}+4 k\right) h\right] \\
& \text { or } \\
= & (-1)^{n}\left[\frac{s_{1} \alpha^{2}-s_{2} \beta^{2}}{k^{2}+4}+\left(H F_{k, 1}\right)^{2}\right] .
\end{aligned}
$$

where $s_{1}=\left(\hat{\alpha} \hat{\beta}-\hat{\alpha}^{2}\right), \quad s_{2}=-\left(\hat{\alpha} \hat{\beta}-\hat{\beta}^{2}\right)$.

Proof: (22): By using (4) we get

$$
\begin{aligned}
& H F_{k, n-1} H F_{k, n+1}-\left(H F_{k, n}\right)^{2} \\
& =\left[\left(F_{k, n-1} F_{k, n+1}-F_{k, n}^{2}\right)-\left(F_{k, n} F_{k, n+2}-F_{k, n+1}^{2}\right)\right. \\
& \left.\quad+\left(F_{k, n} F_{k, n+3}-F_{k, n+1} F_{k, n+2}\right)+\left(F_{k, n+2} F_{k, n+4}-F_{k, n+3}^{2}\right)\right] \\
& +i\left[\left(F_{k, n-1} F_{k, n+2}-F_{k, n} F_{k, n+1}\right)+\left(F_{k, n+2} F_{k, n+2}-F_{k, n} F_{k, n+4}\right)\right] \\
& +\varepsilon\left[\left(F_{k, n-1} F_{k, n+3}-F_{k, n} F_{k, n+2}\right)-\left(F_{k, n} F_{k, n+4}-F_{k, n+2} F_{k, n+2}\right)\right. \\
& \left.\quad+\left(F_{k, n+1} F_{k, n+1}-F_{k, n+2} F_{k, n}\right)+\left(F_{k, n+1} F_{k, n+4}-F_{k, n+2} F_{k, n+3}\right)\right] \\
& + \\
& +h\left(F_{k, n-1} F_{k, n+4}-F_{k, n} F_{k, n+3}\right) \\
& =(-1)^{n}\left[(1-k)+k(1-k) i+(2-k) \varepsilon+\left(k^{3}+4 k\right) h\right],
\end{aligned}
$$

where the identities of the $k$-Fibonacci numbers $F_{k, n-1} F_{k, n+1}-F_{k, n}^{2}=(-1)^{n}[5]$.

We calculate (22) again with a second method. By using (20) we get

$$
\begin{aligned}
H F_{k, n-1} H F_{k, n+1}-\left(H F_{k, n}\right)^{2} & =\left(\frac{\hat{\alpha} \alpha^{n-1}-\hat{\beta} \beta^{n-1}}{\alpha-\beta}\right)\left(\frac{\hat{\alpha} \alpha^{n+1}-\hat{\beta} \beta^{n+1}}{\alpha-\beta}\right)-\left(\frac{\hat{\alpha} \alpha^{n}-\hat{\beta} \beta^{n}}{\alpha-\beta}\right)^{2} \\
& =\frac{\hat{\alpha}^{2} \alpha^{2 n}+\hat{\beta}^{2} \beta^{2 n}-\hat{\alpha} \hat{\beta}(\alpha \beta)^{n}\left(\frac{\beta}{\alpha}\right)-\hat{\beta} \hat{\alpha}(\alpha \beta)^{n}\left(\frac{\beta}{\alpha}\right)}{(\alpha-\beta)^{2}} \\
& -\left(\frac{\hat{\alpha}^{2} \alpha^{2 n}+\hat{\beta}^{2} \beta^{2 n}-2 \hat{\alpha} \hat{\beta}(\alpha \beta)^{n}}{(\alpha-\beta)^{2}}\right) \\
& =(\alpha \beta)^{n-1}\left[\frac{-\hat{\alpha} \hat{\beta} \beta^{2}-\hat{\beta} \hat{\alpha} \alpha^{2}+2 \hat{\alpha} \hat{\beta}(\alpha \beta)}{(\alpha-\beta)^{2}}\right] \\
& =(\alpha \beta)^{n-1}\left[\frac{\left(-\hat{\beta}^{2}\left(\hat{\alpha} \hat{\beta}-\hat{\beta}^{2}\right)-\alpha^{2}\left(\hat{\alpha} \hat{\beta}-\hat{\alpha}^{2}\right)\right.}{(\alpha-\beta)^{2}}-\left(\frac{\hat{\alpha} \alpha-\hat{\beta} \beta}{\alpha-\beta}\right)^{2}\right] \\
& =(-1)^{n}\left[\frac{s_{1} \alpha^{2}-s_{2} \beta^{2}}{k^{2}+4}+\left(H F_{k, 1}\right)^{2}\right],
\end{aligned}
$$

where $s_{1}=\left(\hat{\alpha} \hat{\beta}-\hat{\alpha}^{2}\right), s_{2}=-\left(\hat{\alpha} \hat{\beta}-\hat{\beta}^{2}\right)$ and $\hat{\alpha}=1+i \alpha+\varepsilon \alpha^{2}+h \alpha^{3},-\hat{\beta}=1+i \beta+\varepsilon \beta^{2}+h \beta^{3}$. This completes the proof. 
Theorem 7 (Catalan's Identity). Let $H F_{k, n+r}$ be the $k$-Fibonacci hybrid number. For $n \geq 1$, Catalan's identity for $H F_{k, n+r}$ is as follows:

$$
\begin{aligned}
H F_{k, n+r-1} H F_{k, n+r+1}-\left(H F_{k, n+r}\right)^{2}= & (-1)^{n+r}[(1-k)+k(1-k) i \\
& \left.+(2-k) \varepsilon+\left(k^{3}+4 k\right) h\right] \\
\text { or } \quad & \\
= & (-1)^{n+r}\left[\frac{s_{1} \alpha^{2}-s_{2} \beta^{2}}{k^{2}+4}+\left(H F_{k, 1}\right)^{2}\right] .
\end{aligned}
$$

where $s_{1}=\left(\hat{\alpha} \hat{\beta}-\hat{\alpha}^{2}\right), \quad s_{2}=-\left(\hat{\alpha} \hat{\beta}-\hat{\beta}^{2}\right)$.

Proof: (23) By using (4) we get

$$
\begin{aligned}
& H F_{k, n+r-1} H F_{k, n+r+1}-\left(H F_{k, n+r}\right)^{2} \\
& =\left[\left(F_{k, n+r-1} F_{k, n+r+1}-F_{k, n+r}^{2}\right)-\left(F_{k, n+r} F_{k, n+r+2}-F_{k, n+r+1}^{2}\right)\right. \\
& \left.\quad+\left(F_{k, n+r} F_{k, n+r+3}-F_{k, n+r+1} F_{k, n+r+2}\right)+\left(F_{k, n+r+2} F_{k, n+r+4}-F_{k, n+r+3}^{2}\right)\right] \\
& +i\left[\left(F_{k, n+r-1} F_{k, n+r+2}\right)-\left(F_{k, n+r} F_{k, n+r+1}\right)-\left(F_{k, n+r+1} F_{k, n+r+4}-F_{k, n+r+1} F_{k, n+r+3}\right)\right. \\
& \left.\quad-\left(F_{k, n+r+2} F_{k, n+r+2}-F_{k, n+r+3} F_{k, n+r+1}\right)\right] \\
& +\varepsilon \\
& \quad\left[\left(F_{k, n+r-1} F_{k, n+r+3}-F_{k, n+r} F_{k, n+r+2}\right)+\left(F_{k, n+r} F_{k, n+r+4}-F_{k, n+r+1} F_{k, n+r+3}\right)\right. \\
& \quad+\left(F_{k, n+r+1} F_{k, n+r+1}-F_{k, n+r+2} F_{k, n+r}\right)-\left(F_{k, n+r+1} F_{k, n+r+4}-F_{k, n+r+2} F_{k, n+r+3}\right) \\
& \left.\quad-\left(F_{k, n+r+2} F_{k, n+r+2}-F_{k, n+r+3} F_{k, n+r+1}\right)\right] \\
& +h\left[\left(F_{k, n+r-1} F_{k, n+r+4}-F_{k, n+r} F_{k, n+r+3}\right)-\left(F_{k, n+r} F_{k, n+r+3}-F_{k, n+r+1} F_{k, n+r+2}\right)\right. \\
& \left.\quad+\left(F_{k, n+r+2} F_{k, n+r+1}-F_{k, n+r+3} F_{k, n+r}\right)\right] \\
& =(-1)^{n+r}\left[(1-k)+k(1-k) i+(2-k) \varepsilon+\left(k^{3}+4 k\right) h\right],
\end{aligned}
$$

where the identity of the $k$-Fibonacci numbers $F_{k, n+r-1} F_{k, n+r+1}-F_{k, n+r}^{2}=(-1)^{n+r}$ is used [5]. We calculate (23) again with a second method. By using (20) we get

$$
\begin{aligned}
H F_{k, n+r-1} H F_{k, n+r+1}-\left(H F_{k, n+r}\right)^{2}= & \begin{array}{c}
\left.\frac{\hat{\alpha} \alpha^{n+r-1}-\hat{\beta} \beta^{n+r-1}}{\alpha-\beta}\right)\left(\frac{\hat{\alpha} \alpha^{n+r+1}-\hat{\beta} \beta^{n+r+1}}{\alpha-\beta}\right) \\
-\left(\frac{\hat{\alpha} \alpha^{n+r}-\hat{\beta} \beta^{n+r}}{\alpha-\beta}\right)^{2}
\end{array} \\
= & \frac{\hat{\alpha}^{2} \alpha^{2(n+r)}+\hat{\beta}^{2} \beta^{2(n+r)}-\hat{\alpha} \hat{\beta}(\alpha \beta)^{n+r}\left(\frac{\beta}{\alpha}\right)-\hat{\beta} \hat{\alpha}(\alpha \beta)^{n+r}\left(\frac{\alpha}{\beta}\right)}{(\alpha-\beta)^{2}} \\
= & -\left(\frac{\hat{\alpha}^{2} \alpha^{2(n+r)}+\hat{\beta}^{2} \beta^{2(n+r)}-2 \hat{\alpha} \hat{\beta}(\alpha \beta)^{n+r}}{(\alpha-\beta)^{2}}\right) \\
= & (\alpha \beta)^{n+r-1}\left[\frac{\hat{\alpha} \hat{\beta} \beta^{2}-\hat{\beta} \hat{\alpha} \alpha^{2}+2 \hat{\alpha} \hat{\beta}(\alpha \beta)}{(\alpha-\beta)^{2}}\right] \\
& -\left(\frac{-\hat{\alpha}^{2}\left(\hat{\alpha} \hat{\beta}-\hat{\beta}-\hat{\beta}^{2}\right)-\alpha^{2}\left(\hat{\alpha} \hat{\beta}-\hat{\alpha}^{2}\right)}{(\alpha-\beta)^{2}}\right) \\
= & (-1)^{n+r}\left[\frac{s_{1} \alpha^{2}-s_{2} \beta^{2}}{k^{2}+4}+\left(H F_{k, 1}\right)^{2}\right]
\end{aligned}
$$

where $s_{1}=\left(\hat{\alpha} \hat{\beta}-\hat{\alpha}^{2}\right), s_{2}=-\left(\hat{\alpha} \hat{\beta}-\hat{\beta}^{2}\right)$ and $\hat{\alpha}=1+i \alpha+\varepsilon \alpha^{2}+h \alpha^{3},-\hat{\beta}=1+i \beta+\varepsilon \beta^{2}+h \beta^{3}$. This completes the proof. 


\section{The matrix of $k$-Fibonacci hybrid numbers}

Using the norm of $k$-Fibonacci hybrid number, a $2 \times 2$ matrix representation as follows:

$$
\begin{gathered}
\left\|H F_{k, n}\right\|^{2}=F_{k, n}^{2}+F_{k, n+1}^{2}-F_{k, n+3}^{2}-2 F_{k, n+1} F_{k, n+2} \\
=C\left(H F_{k, n}\right) \\
=\operatorname{det}\left(A_{H F_{k, n}}\right), \\
A_{H F_{k, n}}=\left(\begin{array}{cc}
F_{k, n}+F_{k, n+2} & F_{k, n+1}-F_{k, n+2}+F_{k, n+3} \\
F_{k, n+2}-F_{k, n+1}+F_{k, n+3} & F_{k, n}-F_{k, n+2}
\end{array}\right)
\end{gathered}
$$

Therefore, there is an isomorphic representation from $H F_{k, n}$ to the set of all $M_{2 \times 2}$ matrices can be defined as follows:

$$
\phi: H F_{k, n} \rightarrow M_{2 \times 2}
$$

This transformation is one-to-one and onto. Thus, $H F_{k, n}$ and $M_{2 \times 2}$ are isomorphic.

Using the representations of hybrid numbers in [2], we can give $4 \times 4$ left and right representations of the $k$-Fibonacci hybrid numbers.

$4 \times 4$ left matrix representation of $k$-Fibonacci hybrid number $H F_{k, n}$ is given by

$$
\begin{gathered}
\mathcal{L}_{H F_{k, n}}: H F_{k, n} \rightarrow M_{(4, R)} \\
\mathcal{L}_{H F_{k, n}}=\left(\begin{array}{cccc}
F_{k, n} & F_{k, n+2}-F_{k, n+1} & F_{k, n+1} & F_{k, n+3} \\
F_{k, n+1} & F_{k, n}-F_{k, n+3} & 0 & F_{k, n+1} \\
F_{k, n+2} & -F_{k, n+3} & F_{k, n}+F_{k, n+3} & F_{k, n+1}-F_{k, n+2} \\
F_{k, n+3} & F_{k, n+2} & -F_{k, n+1} & F_{k, n}
\end{array}\right)
\end{gathered}
$$

and $4 \times 4$ the right matrix representation of $k$-Fibonacci hybrid number $H F_{k, n}$ is given by

$$
\begin{gathered}
\mathcal{R}_{H F_{k, n}}: H F_{k, n} \rightarrow M_{(4, R)} \\
\mathcal{R}_{H F_{k, n}}=\left(\begin{array}{cccc}
F_{k, n} & F_{k, n+2}-F_{k, n+1} & F_{k, n+1} & F_{k, n+3} \\
F_{k, n+1} & F_{k, n}+F_{k, n+3} & 0 & -F_{k, n+1} \\
F_{k, n+2} & F_{k, n+3} & F_{k, n}-F_{k, n+3} & F_{k, n+2}-F_{k, n+1} \\
F_{k, n+3} & -F_{k, n+2} & F_{k, n+1} & F_{k, n}
\end{array}\right) .
\end{gathered}
$$

Also, we have $\operatorname{det} \mathcal{L}_{H F_{k, n}}=\operatorname{det} \mathcal{R}_{H F_{k, n}}=\left\|H F_{k, n}\right\|^{4}$.

\section{Conclusion}

In this paper, we have examined the $k$-Fibonacci hybrid numbers, which are non-commutative. Thus, we have derived several interesting properties of the $k$-Fibonacci hybrid numbers such as Honsberger identity, Binet's formula, generating functions, d'Ocagne's identity, Cassini's and Catalan's identities. Furthermore, the $k$-Fibonacci hybrid matrix was defined by obtaining the $2 \times 2$ matrix representation of the $k$-Fibonacci hybrid number $H F_{k, n}$. We also give $4 \times 4$ matrix representations of the $k$-Fibonacci hybrid numbers $H F_{k, n}$. 


\section{Acknowledgements}

The author would like to thank the anonymous reviewers for their valuable comments and suggestions to improve the quality of the paper.

\section{References}

[1] Aydın Torunbalc1, F. (2018). The $k$-Fibonacci Dual Quaternions. International Journal of Mathematical Analysis, 12(8), 363-373.

[2] Çakır, H., \& Özdemir, M. (2021). Hybrid Number Matrices. researchgate.net.

[3] Cerda-Morales, G. (2018). Investigation of Generalized Hybrid Fibonacci Numbers and Their Properties, ArXiv:1806.02231v1.

[4] Falcon, S., \& Plaza, A. (2007). On the Fibonacci $k$-numbers. Chaos Solitons Fractals, 32(5), 1615-1624.

[5] Falcon, S., \& Plaza, A. (2007). The $k$-Fibonacci sequence and the Pascal 2-triangle. Chaos Solitons Fractals, 33(1), 38-49.

[6] Falcon, S. (2011). On the $k$-Lucas numbers. International Journal of Contemporary Mathematical Sciences, 6(21), 1039-1050.

[7] Falcon, S. (2016). On the powers of the $k$-Fibonacci numbers. Ars Combinatoria, 127, 329-338.

[8] Falcon, S. (2016). On the complex $k$-Fibonacci numbers. Cogent Mathematics, 3(1), Article 1201944.

[9] Özdemir, M. (2018). Introduction to Hybrid Numbers. Advances in Applied Clifford Algebras, 28(11), 10-32.

[10] Polatlı, E., Kızılateş, C., \& Kesim, S. (2016). On Split $k$-Fibonacci and $k$-Lucas Quaternions. Advances in Applied Clifford Algebras, 26(1), 353-362.

[11] Ramirez, J. L. (2015). Some combinatorial properties of the $k$-Fibonacci and the $k$-Lucas quaternions. Analele Universitatii Ovidius Constanta-Seria Matematica, 23(2), 201-212. 